\title{
Cobalt-protoporphyrin improves heart function by blunting oxidative stress and restoring NO synthase equilibrium in an animal model of experimental diabetes
}

\section{Jian Cao ${ }^{1,2 \dagger}$, Cecilia Vecoli ${ }^{3,4}{ }^{\dagger}$, Danilo Neglia ${ }^{5}$, Barbara Tavazzi $^{6}$, Giuseppe Lazzarino ${ }^{7}$, Michela Novelli $^{8}$, Pellegrino Masiello ${ }^{8}$, Yu-tang Wang ${ }^{1}$, Nitin Puri ${ }^{2}$, Nazareno Paolocci ${ }^{4,9}$, Antonio L'Abbate ${ }^{3,5 t}$ and Nader G. Abraham $^{2 * t}$}

${ }^{1}$ First Geriatric Cardiology Division, Chinese PLA General Hospital, Beijing, China

${ }^{2}$ Department of Physiology and Pharmacology, University of Toledo, Toledo, OH, USA

${ }^{3}$ Scuola Superiore Sant'Anna, Pisa, Italy

${ }^{4}$ Division of Cardiology, Johns Hopkins University, Baltimore, MD, USA

${ }^{5}$ CNR Institute of Clinical Physiology, Pisa, Italy

${ }^{6}$ Catholic University of Rome "Sacro Cuore," Rome, Italy

7 Department of Biology, Geology and Environmental Sciences, Division of Biochemistry and Molecular Biology, University of Catania, Catania, Italy

${ }^{8}$ Department of Experimental Pathology, University of Pisa, Pisa, Italy

${ }^{9}$ Department of Clinical Medicine, University of Perugia, Perugia, Italy

\section{Edited by:}

Francesco Visioli, Madrid Institute for Advanced Studies - Food, Spain

Reviewed by:

Maria Alvarez De Sotomayor,

Universidad de Sevilla, Spain

Shane Thomas, University of New

South Wales, Australia

\section{*Correspondence:}

Nader G. Abraham, Department of

Physiology and Pharmacology,

University of Toledo, Toledo, $\mathrm{OH}$

43614, USA.

e-mail:nader.abraham@utoledo.edu

${ }^{+}$Jian Cao and Cecilia Vecoli contributed equally to this work.

Nader G. Abraham and Antonio

L'Abbate are equally senior authors.
Myocardial dysfunction and coronary macro/microvascular alterations are the hallmarks of diabetic cardiomyopathy and are ascribed to increased oxidative stress and altered nitric oxide synthase (NOS) activity. We hypothesize that pre-treatment by cobalt-protoporphyrin IX (CoPP) ameliorates both myocardial function and coronary circulation in streptozotocin (STZ)-induced diabetic rats. Isolated hearts from diabetic rats in Langendorff configuration displayed lower left ventricular function and higher coronary resistance (CR) compared to hearts from control animals. CoPP treatment of diabetic animals $(0.3 \mathrm{mg} / 100 \mathrm{~g}$ body weight i.p., once a week for 3 weeks) significantly increased all the contractile/relaxation indexes $(p<0.01)$, while decreasing CR $(p<0.01)$. CoPP enhanced HO-1 protein levels and reduced oxidative stress in diabetic animals, as indicated by the significant $(p<0.05)$ decrease in heart \% GSSG, $\mathrm{O}_{2}^{-}$, and malondialdehyde (MDA) levels. CoPP increased adiponectin levels and phosphorylation of AKT and AMPK and reversed the eNOS/iNOS expression imbalance observed in the untreated diabetic heart. Furthermore, after CoPP treatment, a rise in malonyl-CoA as well as a decrease in acetyl-CoA was observed in diabetic hearts. In this experimental model of diabetic cardiomyopathy, CoPP treatment improved both cardiac function and coronary flow by blunting oxidative stress, restoring eNOS/iNOS expression balance and increasing $\mathrm{HO}-1$ levels, thereby favoring improvement in both endothelial function and insulin sensitivity.

Keywords: diabetes, cardiac contractility, AMPK, nitric oxide, coronary microcirculation

\section{INTRODUCTION}

According to World Health Organization, diabetes mellitus is a global pandemic. It is associated with an increasing incidence of heart failure, coronary artery disease, and cardiovascular mortality (LeWinter, 1996; Adeghate, 2004). The mechanisms responsible for the effects of hyperglycemia and diabetes on myocardial function and on coronary macro- and micro-vasculature are the object of intensive investigation. Among several putative mechanisms, the role of the reactive oxygen (ROS) and reactive nitrogen species (RNS) has been studied in a number of experimental models of diabetes (Stevens, 2005). Oxidative stress associated with deficient antioxidant systems has been reported to play a critical role in subcellular remodeling, calcium handling, and subsequent diabetic cardiomyopathy (Haidara et al., 2006). Nitric oxide (NO) signaling is also involved in the genesis of diabetic myocardial and vascular damage and an altered expression of nitric oxide synthase
(NOS) isoforms occurs in diabetes as well as in the hyperglycemic state (Ceriello et al., 2002; Nagareddy et al., 2005).

The levels of adiponectin, an adipocytokine secreted from the adipose tissue (Chandran et al., 2003), are reduced in diabetes (Hotta et al., 2000), insulin resistance (Weyer et al., 2001), and obesity (Yamauchi et al., 2001). Decreased levels of adiponectin have been proposed to mediate some of the deleterious effects of diabetes on myocardial function at rest as well as in response to ischemia (L'Abbate et al., 2007). Accordingly, administration of adiponectin to adiponectin-deficient knockout mice improved recovery of cardiac function after myocardial ischemia (Tao et al., 2007). Adiponectin has different cardiac effects including the increase in myocardial insulin sensitivity, stimulation of glucose uptake and lipid oxidation, increase in eNOS activity (Tao et al., 2007), and anti-inflammatory action (Antoniades et al., 2009). 
In severe diabetes, myocardial function may be depressed as a result of multiple pathogenic mechanisms (Boudina and Abel, 2007). Impairment of myocardial function is seen in streptozotocin (STZ) treated rats, a model of severe diabetes which reproduces the clinical features of diabetic cardiomyopathy (An and Rodrigues, 2006). However, in this model the interaction between microvascular abnormalities and myocardial function has never been explored. In a previous study performed in mildly diabetic rats obtained by STZ-nicotinamide treatment, we observed a significant increase in microvascular resistance during ischemia/reperfusion, associated with myocardial iNOS overexpression, eNOS downregulation, and increased oxidative stress (L'Abbate et al., 2007). In these diabetic rats, the cobaltprotoporphyrin IX pre-treatment (CoPP) improved the coronary vascular response to ischemia-reperfusion, restoring the balance of cardiac eNOS and iNOS isoforms and the redox state. It is also documented that CoPP treatment increases EC-SOD as well as mitochondrial function in animal models of diabetes (Turkseven et al., 2005; Di Noia et al., 2006). Recently, Kusmic et al. demonstrated that CoPP enhances the crosstalk between pAKTpAMPK, thus increasing NO production and bioavailability in a mouse model of diabetes. This effect of CoPP resulted in both local and systemic effects eventually leading to the restoration of microvascular function in the heart of diabetic mice (Kusmic et al., 2010).

The present study was designed to determine, in a rat model of severe diabetes and cardiomyopathy, whether in vivo CoPP treatment could improve myocardial as well as coronary function. We examined the interaction between cardiac physiological parameters and oxidative stress, focusing in particular on eNOS and iNOS expression and adiponectin signaling modulation.

\section{MATERIALS AND METHODS}

All experiments were approved by the Institutional Animal Care and Use Committee of Johns Hopkins University and conducted under the guidelines for the Care and Use of Laboratory Animals published by the Office of Science and Health Reports, NIH.

\section{INDUCTION OF DIABETES}

Male Wistar rats, 2 months of age, received an injection of $50 \mathrm{mg} / \mathrm{kg}$ streptozotocin (STZ, Sigma, St. Louis, MO, USA), i.p., dissolved in citrate buffer ( $\mathrm{pH} 4.5)$, to obtain a stable diabetes with severely reduced beta-cell mass. STZ-treated animals were matched by a group of controls of the same age receiving the vehicle of STZ.

\section{ADMINISTRATION OF CoPP}

CoPP was purchased from Frontier Scientific, Inc. (Logan, UT, USA). Four weeks after induction of diabetes, CoPP $(0.3 \mathrm{mg} / 100 \mathrm{~g}$ body weight), or the corresponding volume of vehicle $(0.1 \mathrm{M}$ sodium citrate buffer, $\mathrm{pH}$ 7.8) was administered subcutaneously once a week for 3 weeks. Glucose levels of diabetic rats were checked before starting of CoPP treatment, so that animals could be homogenously and randomly distributed between the two groups receiving or not CoPP. In total, three groups of animals were studied: control rats $(\mathrm{C}, n=20)$, diabetic rats $(\mathrm{D}, n=16)$, and diabetic rats treated with $\operatorname{CoPP}(\mathrm{D}+\mathrm{CoPP}, n=16)$. At the end of the experimental period, before excision of the heart, blood samples were taken for glucose, insulin, and adiponectin measurements.

\section{ISOLATED HEART PREPARATION}

Three days after the last CoPP or vehicle injections, rats were anesthetized with i.p. pentobarbital (250 units/ $\mathrm{kg}$ ) and heparinized via the left femoral vein. The heart was rapidly excised, placed in cold perfusion medium and weighed. The isolated heart was attached to the Langendorff apparatus and retrogradely perfused (at $37^{\circ} \mathrm{C}$ ) using constant perfusion pressure of $80 \mathrm{~cm} \mathrm{H}_{2} \mathrm{O}$. The perfusion medium consisted of oxygenated Krebs-Henseleit buffer (L'Abbate et al., 2007). After 20 min of stabilization, cardiac and coronary functions were monitored. For measurement of left ventricular systolic (LVP) and diastolic (EDP) pressure, a latex balloon was inserted into the left ventricle through the mitral valve and connected to a Harvard pressure transducer. In each experiment, balloon volume, and thus left ventricular volume, was adjusted according to an EDP value of $10 \mathrm{mmHg}$ and maintained constant for the entire duration of the experiment. For a constant left ventricular volume and in absence of any ejection (isometric contraction), changes in systolic pressure and $\mathrm{dP} / \mathrm{d} t_{\max }$ are positively correlated to myocardial contractility (inotropism), while changes in $\mathrm{dP} / \mathrm{d} t_{\min }$ are positively correlated to ventricular relaxation. Coronary perfusion pressure (CPP) was monitored by a second pressure transducer connected to the aortic cannula. Data were acquired using a BIOPAC 100 System and analyzed with AcqKnowledge software (BIOPAC system). Left ventricular developed pressure $(\mathrm{LVDevP}=\mathrm{LVP}-\mathrm{EDP})$, heart rate $(\mathrm{HR}), \mathrm{dP} / \mathrm{d} t_{\max }$, $\mathrm{dP} / \mathrm{d} t_{\min }$, and CPP were all derived or calculated from the continuous monitoring of the LV pressure signal while LVP $\times \mathrm{HR}$ defined the rate-pressure product (RPP), an index of cardiac oxygen consumption and energy production.

In all the experiments, coronary flow $(\mathrm{ml} / \mathrm{min})$ was continuously monitored by collecting the cardiac effluent. Coronary resistance (CR) was defined as CPP divided by coronary flow/g of myocardial tissue $(\mathrm{mmHg} \mathrm{min} \mathrm{g} / \mathrm{ml})$.

\section{BLOOD MEASUREMENTS OF GLUCOSE, INSULIN, AND ADIPONECTIN}

Tail vein blood samples were collected from animals fasting for at least $6 \mathrm{~h}$. Glucose levels were measured with a commercial Glucose Monitor Kit (Ascensia Contour Monitoring System, Bayer) and plasma insulin content with Rat Insulin Enzyme Immunoassay kit (Cayman). Plasma adiponectin was determined using an ELISA assay (Pierce Biotechnology, Woburn, MA, USA).

\section{TISSUE PREPARATION AND MEASUREMENT OF MALONDIALDEHYDE (MDA), REDUCED AND OXIDIZED GLUTATHIONE, MALONYL-COA, AND ACETYL-CoA}

Each left ventricular tissue sample was processed according to an organic deproteinization procedure suitable for the determination of water-soluble low-molecular weight compounds representative of both tissue oxido-reductive and energy status. Malondialdehyde (MDA), reduced and oxidized glutathione (GSH and GSSG respectively) malonyl-CoA, and acetyl-CoA were measured by ion-pairing HPLC (Lazzarino et al., 2003). Data acquisition and analysis were performed using the ChromQuest software package (Thermo Electron Corporation, Waltham, MA, USA). 


\section{WESTERN BLOT ANALYSIS OF CARDIAC SIGNALING MOLECULES AND HO ACTIVITY LEVEL}

Frozen hearts were pulverized under liquid nitrogen and placed in a homogenization buffer $(10 \mathrm{mM}$ phosphate buffer, $250 \mathrm{mM}$ sucrose, $1 \mathrm{mM}$ EDTA, $0.1 \mathrm{mM}$ PMSF, and $0.1 \%$ tergitol, $\mathrm{pH}$ 7.5) and used for measuring signaling molecules as previously described (L'Abbate et al., 2007). Protein levels were visualized by immunoblotting with antibodies against HO-1, HO-2 (Stressgen Biotechnologies Corp., Victoria, BC, Canada). Antibodies against Akt, pAkt, AMPK, pAMPK, and adiponectin were obtained from Cell Signaling Technology, Inc. (Beverly, MA, USA). eNOS, iNOS, and p-eNOS from Santa Cruz Biotechnology (Santa Cruz, CA, USA). Briefly, $20 \mathrm{mg}$ of heart tissue lysate supernatant was separated by $12 \%$ SDS/polyacrylamide gel electrophoresis and transferred to a nitrocellulose membrane. Chemiluminescence detection was performed with the Amersham ECL detection kit (Amersham, Piscataway, NJ, USA), according to the manufacturer's instructions. The image was analyzed by densitometry. Protein bands were quantified and values were normalized to those of actin. One out of three separate experiments with consistent results is shown.

HO-1 activity was assess using a scanning double beam spectrophotometer (Lambda 17 UV/Vis; Perkin Elmer Cetus Instruments, Norfolk, CT, USA) and expressed as nmol bilirubin/mg protein/h (L'Abbate et al., 2007).

\section{SUPEROXIDE CARDIAC TISSUE LEVELS}

Superoxide $\left(\mathrm{O}_{2}^{-}\right)$was determined placing hearts in plastic scintillation minivials, containing $5 \mu \mathrm{M}$ lucigenin for the detection of $\mathrm{O}_{2}^{-}$ as previously described (L'Abbate et al., 2007). Briefly, cardiac tissues were placed in plastic scintillation minivials containing $5 \mu \mathrm{M}$ lucigenin in $1 \mathrm{ml}$ of Krebs solution buffered with $10 \mathrm{mM}$ HEPES$\mathrm{NaOH}$ ( $\mathrm{pH}$ 7.4). The chemiluminescence from superoxide was measured by a liquid scintillation counter (LS6000IC; Beckman Instruments, San Diego, CA, USA) with a single active photomultiplier tube in a dark room. Background chemiluminescence in the absence of tissue was subtracted from subsequent measurements made in the presence of BPA. The tissue was weighed at the end of the experiment, and the counts were divided by weight to give the readings in counts/min/mg of tissue (L'Abbate et al., 2007).

\section{STATISTICAL ANALYSIS}

Results are presented as mean \pm standard error of the mean (SEM) of the number $(n)$ of replicate determinations. Statistical significance between experimental groups and between different study conditions was determined by using a two-way ANOVA, followed by the Fisher's exact test to evaluate two-by-two differences. Comparison of the three groups was determined first doing an ANOVA followed by a post hoc test. $p<0.05$ was considered significant.

\section{RESULTS \\ COPP TREATMENT IMPROVES INSULIN SENSITIVITY AND INCREASES PLASMA ADIPONECTIN LEVELS}

At the end of the experimental period, plasma glucose levels in diabetic (D) rats were $463.4 \pm 19.1 \mathrm{mg} / \mathrm{dl}$ compared to $119 \pm 2.6 \mathrm{mg} / \mathrm{dl}$ in control $(\mathrm{C})$ group $(p<0.01)$ and the corresponding plasma insulin levels were $0.87 \pm 0.22 \mathrm{ng} / \mathrm{ml}$ compared to $1.9 \pm 0.35 \mathrm{ng} / \mathrm{ml}(p<0.01)$ respectively. CoPP treatment lowered glucose levels in diabetic rats to $386.7 \pm 18 \mathrm{mg} / \mathrm{dl}(p<0.05$ vs. D) without changes in insulin levels $(0.86 \pm 0.35 \mathrm{ng} / \mathrm{ml})$, thus increasing insulin sensitivity. Plasma adiponectin levels were significantly lower in diabetic rats compared to controls $(3.06 \pm 1.08$ vs. $8.05 \pm 0.9 \mu \mathrm{g} / \mathrm{ml} ; p<0.05)$. The administration of CoPP to diabetic rats resulted in a marked increase in plasma adiponectin concentration $(11 \pm 1.9 \mu \mathrm{g} / \mathrm{ml}, p<0.01$ vs. D).

\section{CoPP TREATMENT IMPROVES CARDIAC AND MICROVASCULAR FUNCTION}

After 20 min of stabilization, hearts isolated from diabetic animals exhibited increased CR and depressed cardiac function as compared with hearts isolated from control animals (Figure 1; Table 1). CoPP treatment increased RPP in diabetic hearts $(p<0.005)$. The improvement of RPP in diabetic hearts was the result of a combined effect of CoPP on the inotropic and chronotropic features of the heart. Indeed, systolic LVP, LVDevP, and $\mathrm{dP} / \mathrm{d} t_{\max }$ (all related to inotropism) were increased in $\mathrm{D}+\mathrm{CoPP}$ compared to $\mathrm{D}(p<0.005)$. Likewise, heart rate was higher in D + CoPP hearts as compared to D (Table 1). Moreover, CoPP improved cardiac relaxation in D hearts $(p<0.005)$ at values similar to that found in control animals. When coronary microcirculation was examined, it was found that CR was higher in $\mathrm{D}$ as compared to $\mathrm{C}$ hearts $(p<0.01)$ and returned back to control values after CoPP pre-treatment (Figure 1).

\section{EFFECT OF COPP TREATMENT ON HO-1 AND HO-2 EXPRESSION AND HO ACTIVITY}

Diabetic hearts displayed lower HO-1 protein levels and lower $\mathrm{HO}$ activity ( $p<0.05$ vs. C) compared to those found in control animals (Figures 2A,B). CoPP treatment caused an increase in both $\mathrm{HO}-1$ protein levels and $\mathrm{HO}$ activity in diabetic animals $(p<0.01$ vs. D; Figure 2). Both the diabetic condition and pre-treatment with CoPP did not influence the levels of HO-2 protein (upper left panel).

\section{EFFECT OF CoPP TREATMENT ON GLUTATHIONE, MDA, $0_{2}^{-}$AND 3-NT}

When compared to control, heart tissue in D rats showed a higher value of \% GSSG [GSSG/GSH+GSSG) × 100], most likely dependent on a shift of redox balance towards an oxidative state. This value went back to a value close to that of controls after CoPP administration $(p<0.01$; Figure 3$)$. Moreover, a similar trend in the GSH balance was also found by considering the GSH/GSSG ratio ( $\mathrm{C}$ rats $=16.98$; $\mathrm{D}$ rats $=8.17$; CoPP rats $=11.21$ ). In addition to altered glutathione balance, diabetic hearts exhibited a five-fold increase in $\mathrm{O}_{2}^{-}$levels compared to controls $(p<0.05$ vs. $\mathrm{C})$, that was completely reversed upon CoPP treatment $(p<0.01$ $\mathrm{D}+\mathrm{CoPP}$ vs. D; Figure 3). Compared to controls, diabetic hearts showed remarkably higher levels of MDA, an index of lipid peroxidation ( $p<0.01$ vs. C). In D CoPP rats, both MDA and 3-NT levels found returned toward normal values of control animals, probably as a consequence of HO-1 induction (Figure 3).

\section{CoPP TREATMENT INCREASES CARDIAC LEVELS OF eNOS, p-eNOS, AND iNOS}

Consistent with previous observations, we found a lower value of p-eNOS/eNOS ratio in hearts isolated from diabetic animals than 

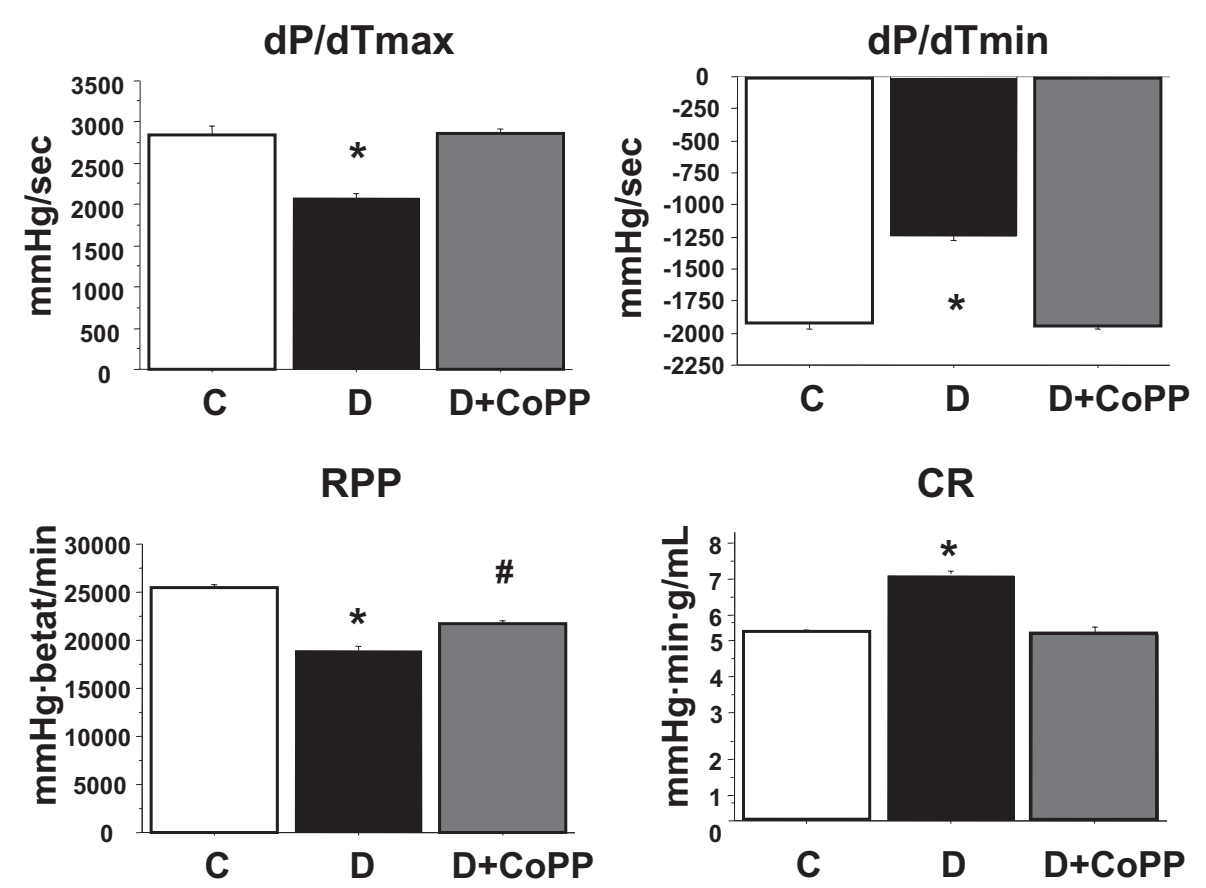

FIGURE 1 | After $30 \mathrm{~min}$ of stabilization, $\mathrm{dP} / \mathrm{d} t_{\max }$, and $\mathrm{dP} / \mathrm{d} t_{\min }$, rate-pressure product (RPP), and levels of coronary resistance (CR) in control rats $(C, n=20)$, diabetic rats $(D, n=15)$, and diabetic rats treated

Table 1 | Hemodynamic parameters in control rats $(C, n=20)$, diabetic rats $(D, n=16)$, and diabetic rats treated for 3 weeks with CoPP (D + CoPP, $n=16$ ).

\begin{tabular}{llcc}
\hline & HR (beats $/ \mathbf{m i n})$ & LVP $(\mathbf{m m H g})$ & LVDP (mmHg) \\
\hline $\mathrm{C}(n=20)$ & $213 \pm 3$ & $120 \pm 6$ & $110 \pm 4$ \\
$\mathrm{D}(n=16)$ & $178 \pm 3^{*}$ & $93 \pm 9^{\#}$ & $81 \pm 5^{\#}$ \\
$\mathrm{D}+\mathrm{CoPP}(n=16)$ & $207 \pm 4$ & $105 \pm 5^{*}$ & $94 \pm 3^{*}$
\end{tabular}

Data are expressed as means \pm SEM. HR, heart rate; LVP, left ventricular systolic pressure; LVDP, left ventricular developed pressure calculated as described in Section "Materials and Methods," * $p<0.05$ vs. other groups, " $p<0.05$ vs. controls.

from controls ( $p<0.05$; Figure 4). CoPP treatment increasing the levels of $\mathrm{p}$-eNOS augmented the $\mathrm{p}$-eNOS/eNOS ratio $(p<0.05$ $\mathrm{D}$ vs. D + CoPP and C; Figure 4). Hearts from diabetic animals showed an increased expression of iNOS, that was significantly attenuated upon CoPP treatment (Figure 4).

\section{CoPP TREATMENT INCREASES CARDIAC LEVELS OF AKT, AMPK, AND ADIPONECTIN}

In the hearts from diabetic animals, a significant $(p<0.005)$ decrease of pAKT and pAMPK levels was observed, as compared to controls, without any change in Akt and AMPK (Figure 5). CoPP fully reversed these changes, normalizing pAKT, and even increasing pAMPK levels in diabetic hearts. As shown in Figure 5 (lower panel), adiponectin levels were significantly decreased in diabetic cardiac tissue, but were restored by CoPP treatment $(p<0.05 \mathrm{D}$ vs. $\mathrm{D}+\mathrm{CoPP})$.

\section{CoPP TREATMENT INCREASES CARDIAC LEVELS OF MALONYL-CoA AND DECREASES LEVELS OF ACETYL-CoA}

Diabetes resulted in a significant decrease in the myocardial levels of malonyl-CoA $(p<0.01)$ associated to a significant increase $(p<0.05)$ of acetyl-CoA (Figure 6). Both these changes were normalized after CoPP treatment.

\section{DISCUSSION}

In the present study we demonstrate that CoPP administration increased expression of HO-1 and reversed both myocardial and coronary dysfunction in the STZ rat model of diabetes. In this model of severe diabetes, myocardial function is reduced and CR is increased (Koch et al., 2003; Boudina and Abel, 2007), thus mimicking the clinical nature of diabetic cardiomyopathy with increased coronary microvascular resistance. We demonstrate for the first time in this report a complete reversal of LV systolic and diastolic dysfunction by treatment with the HO-1 protein inducer CoPP.

Following CoPP treatment, the normalization of myocardial function and the reduction of CR in diabetic hearts were associated with a decrease in oxidative stress and improved cardiac markers of endothelial function. In particular, CoPP treatment resulted in the reversion of overexpression of iNOS found in the diabetic heart and the restoration of eNOS expression. This contrasting effect of CoPP on cardiac eNOS and iNOS, leading to the normalization of the expression balance of the two 

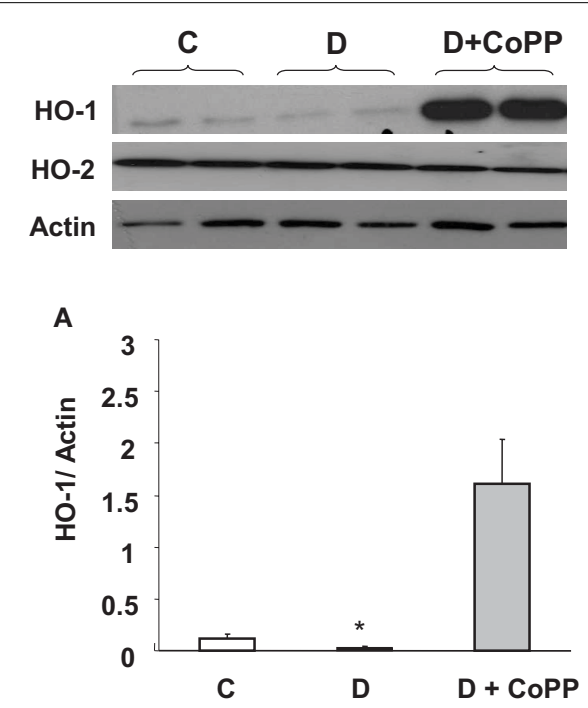

FIGURE 2 | HO-1/HO-2 expression and HO activity in C, D, and

D + CoPP rat hearts. Heart samples were subjected to Western blotting for the determination of $\mathrm{HO}-1 / \mathrm{HO}-2$ protein.

A representative blot is shown. Densitometric analysis of HO-1 vs.

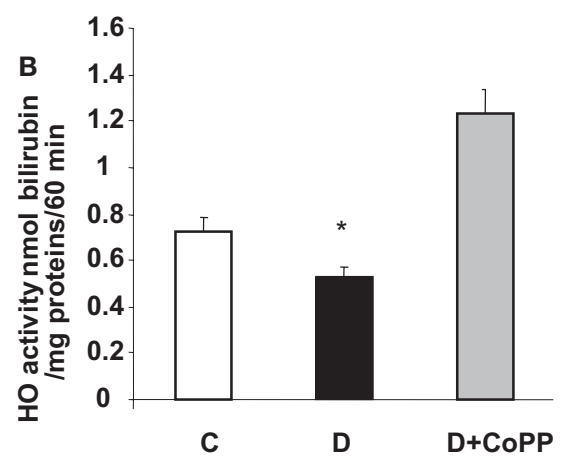

actin is shown and expressed as means \pm SEM of three independent experiments. $\mathrm{HO}$ activity was determined by bilirubin formation (see Materials and Methods). ${ }^{*} p<0.05$ vs. other groups.

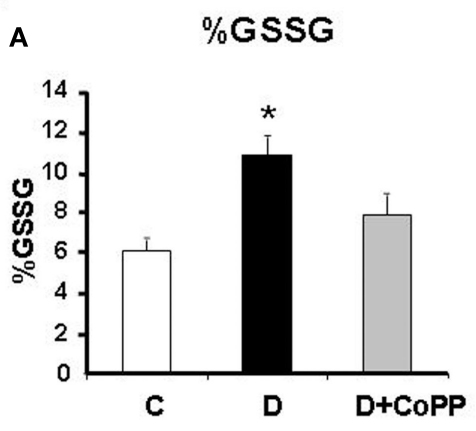

C

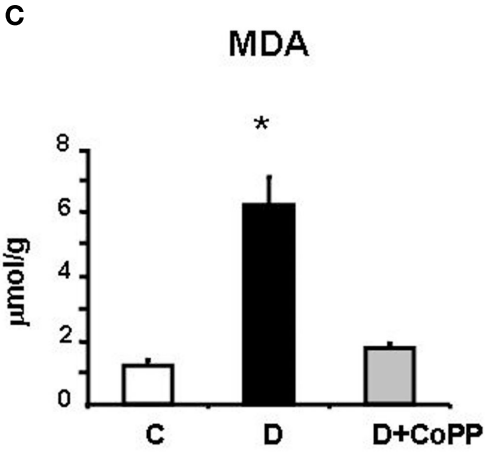

FIGURE 3 | Cardiac tissue levels of (A) \% GSSG

[GSSG/(GSH+GSSG) $\times 100$ ], (B) $\mathrm{O}_{2}^{-}$, and (C) malonyldialdehyde (MDA) measured in hearts from C, D, and D + CoPP rats. (D) Representative

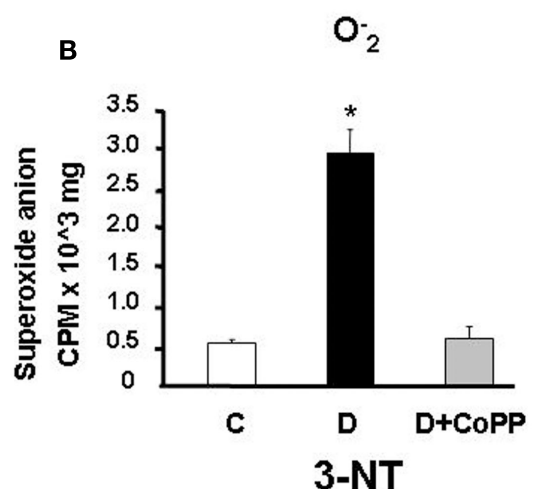

D

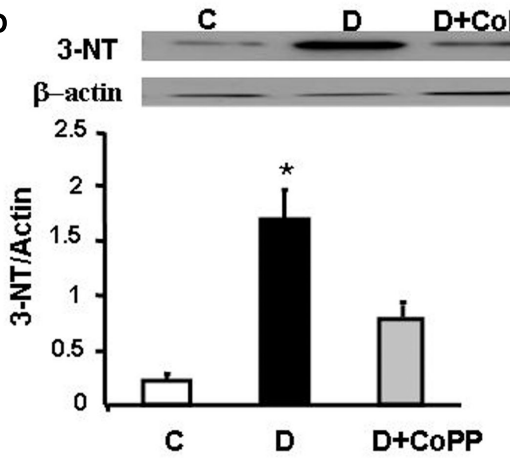

Western blot and densitometric analysis of 3-nitrotyrosine (3-NT) expression in cardiac tissue of $C, D$, and D+CoPP rats. Data are expressed as means $\pm S E M$ of three independent determinations ${ }^{*} p<0.05$ vs. other groups.

It is well known that the beneficial effects of CoPP are mediated by HO-1 induction (Di Noia et al., 2006; Li et al., 2008). In particular, the effect on NOS balance can be ascribed to $\mathrm{HO}-1$, isoforms, could have synergistically contributed to the improvement of both myocardial and vascular functions of the diabetic heart. 

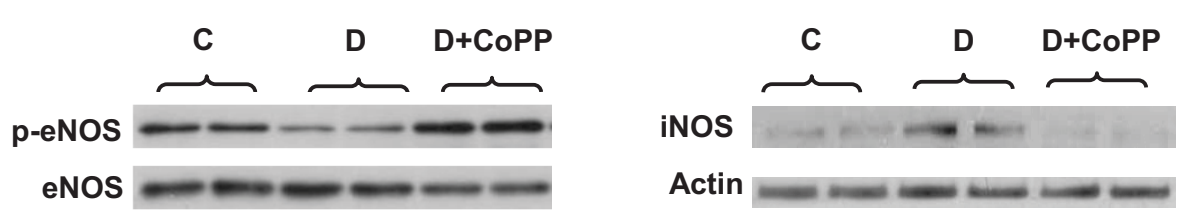

Actin
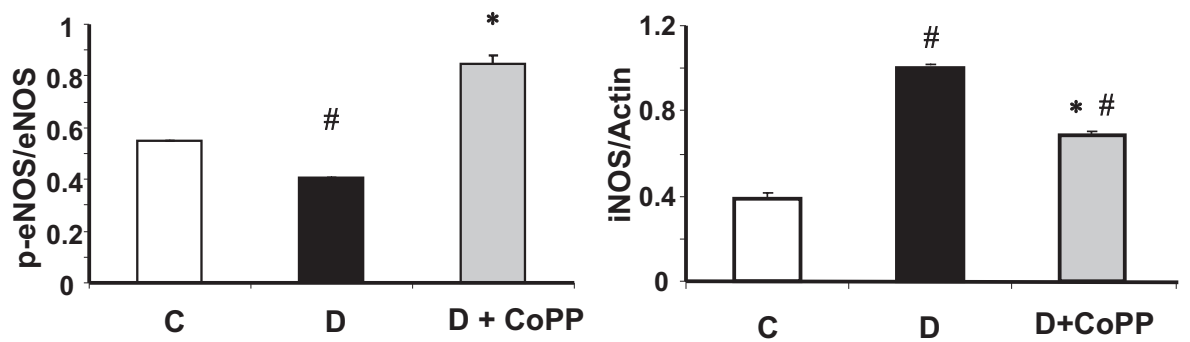

FIGURE 4 | Representative Western blot and densitometric analysis of eNOS, p-eNOS, iNOS, from C, D, and D+CoPP rat hearts.

Quantitative densitometry evaluation of iNOS and p-eNOS/eNOS ratio

from three independent experiments is shown. Data are expressed as means \pm SEM of three independent determinations ${ }^{*} p<0.05$ vs. C; ${ }^{*} p<0.05$ vs. D.
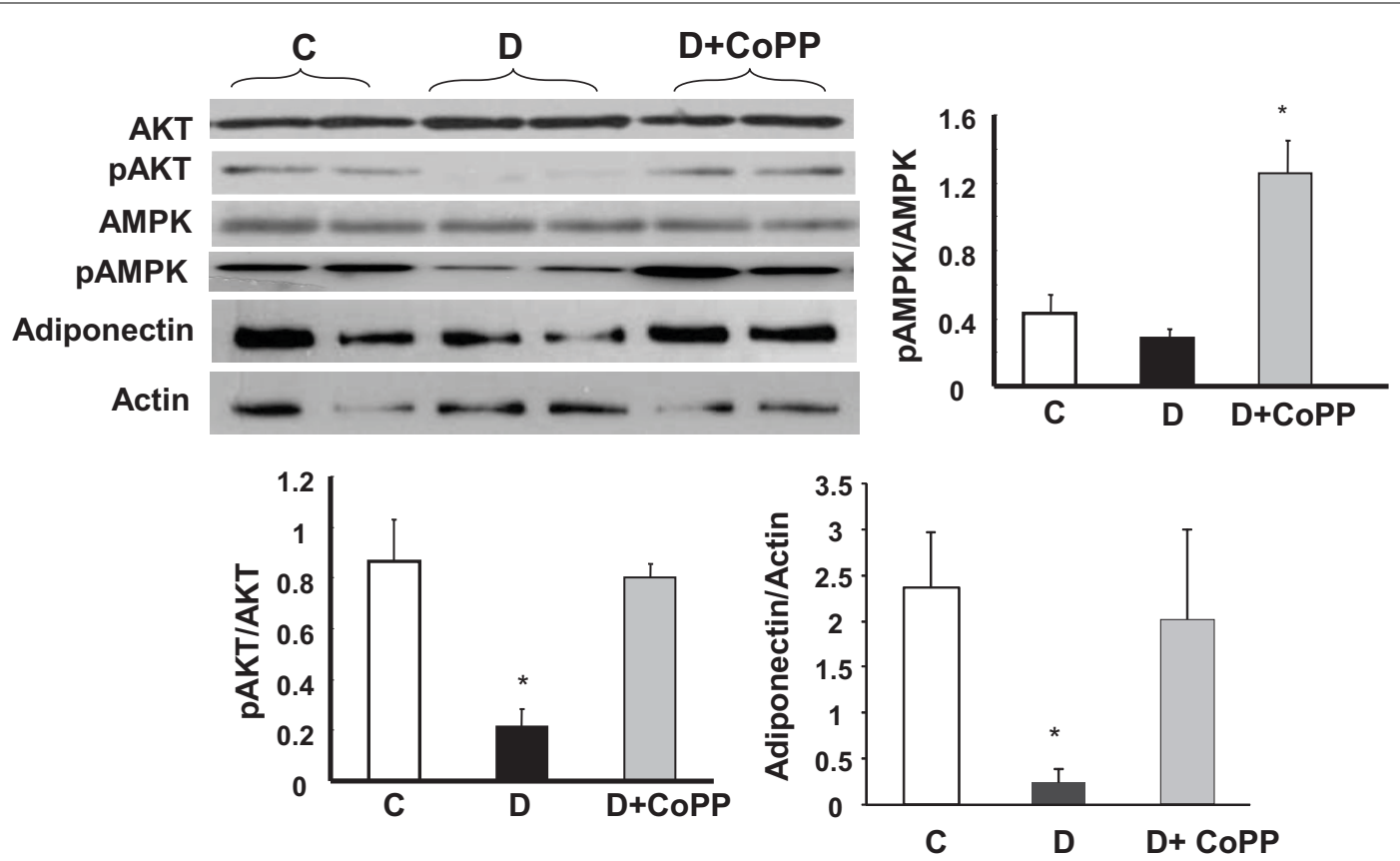

FIGURE 5 | Representative Western blot and densitometric analysis of cardiac expression of pAKt, Akt, pAMPK, AMPK, and adiponectin. Data are expressed as means \pm SEM of three independent determinations of pAkt/Akt and pAMPK/AMPK ratios as well as of adiponectin/actin ratio. ${ }^{*} p<0.05$ vs. other groups; ${ }^{*} p<0.01$ vs. C. since Turkeseven et al. demonstrated that the effect of CoPP was associated with an increase in EC-SOD and NO bioavailability. In contrast, a decrease in $\mathrm{HO}-1$ and inhibition of $\mathrm{HO}$ activity resulted in an increase in superoxide levels in healthy animals (Turkseven et al., 2005).

The improvement of coronary and myocardial function in diabetic animals described here mirror the systemic humoral effects of CoPP that were manifest by the activation of the HO-1/ adiponectin pathway. Previously, we showed the active role of HO-1 in the stimulation of adiponectin secretion. In fact, the inhibition of $\mathrm{HO}$ activity by administration of tin mesoporphyrin (SnMP) after CoPP treatment resulted in decreased levels of plasma adiponectin (L'Abbate et al., 2007). Accordingly, Li et al. (2008) showed that the co-administration of CoPP and SnMP resulted in the loss of the beneficial effects associated with the increased expression of HO-1. The effects of CoPP on increasing the levels of adiponectin were associated with the systemic enhancement of insulin sensitivity, cardiac modulation of glucose uptake, and fatty acid oxidation resulting in improvement in myocardial function. 


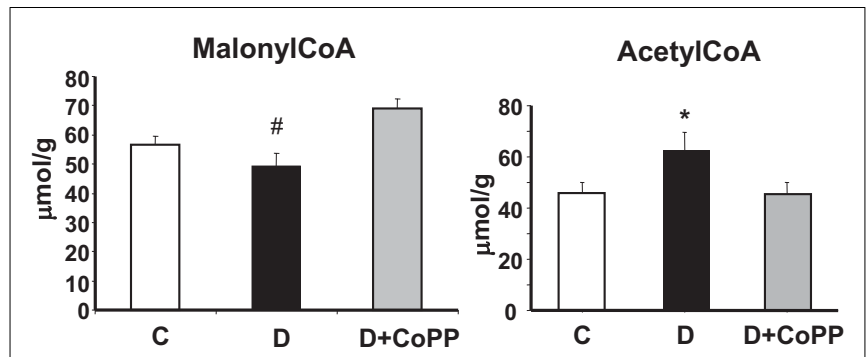

FIGURE 6 | Effect of CoPP on malonyl-CoA and acetyl-CoA in hearts isolated from C, D, and D + CoPP animals. Data are expressed as means \pm SEM of three independent determinations ${ }^{*} p<0.01 \mathrm{D}$ vs. other groups; ${ }^{*} p<0.05$ vs. other groups

Reactive oxygen species have been identified as a primary cause for both the development and progression of diabetic cardiomyopathy (Boudina and Abel, 2007). Indeed, in the present study, the beneficial effects of CoPP on CR and myocardial function in diabetes were associated with a marked reduction of oxidative stress markers in cardiac tissue. CoPP treatment reduced the cardiac levels of superoxide, oxidized glutathione, and MDA. This beneficial effect on redox balance is likely driven by bilirubin, produced from degradation of heme by HO-1, which has strong antioxidant properties as well as cytoprotective effects on the cardiovascular system (Abraham and Kappas, 2008).

The diabetic heart exhibits a progressive decrease in eNOS expression which is accompanied by a concomitant rise in iNOS expression (Bardell and MacLeod, 2001; Boudina and Abel, 2007; L'Abbate et al., 2007). We also found downregulation of eNOS and overexpression of iNOS in the diabetic rat heart, both reversible upon CoPP treatment. Inducible NOS produces a higher amount of NO than other isoforms. In conditions of oxidative stress, such as in the diabetic heart, the overproduction of $\mathrm{NO}$ results in $\mathrm{NO}$ interacting with superoxide and contributing to the formation of RNS including peroxynitrite. Besides reducing NO availability, peroxynitrite is a strong oxidant and can oxidize various biomolecules and exert cytotoxic actions in both myocardial and vascular tissue resulting in cardiovascular derangement. Peroxynitrite formation is associated with several pathophysiological conditions including acute myocardial infarction and chronic ischemic heart failure (Weinstein et al., 2000; Pacher et al., 2003). It contributes to the pathogenesis of diabetic cardiovascular complications while its pharmacological decomposition improves both diastolic and systolic function in diabetic heart (Szabo et al., 2002). Peroxynitrite depresses cardiac function through different mechanisms including cellular necrosis or $\alpha$-actinin nitration and its effective neutralization can have a significant therapeutic benefit.

In addition to decreased iNOS levels and NO production, thus blunting the formation and deleterious effects of abnormal amounts of peroxynitrite, increased levels of p-eNOS were observed following CoPP treatment. This may be due to the CoPPmediated increases in plasma and cardiac tissue adiponectin. Adiponectin differentially regulates NO production from eNOS and iNOS: through crosstalk between AMPK and AKT, it promotes eNOS phosphorylation pathways, increasing eNOS activity (Tao et al., 2007). Recently, it was reported that ventricular cardiomyocytes also express adiponectin receptors leading to increased glucose uptake and fatty acid oxidation in normal rats (Ding et al., 2007) thus improving their metabolic profile (Kim et al., 2007). Human studies demonstrate that plasma adiponectin regulates insulin receptor phosphorylation contributing to increased insulin sensitivity (Yamauchi et al., 2001; Stefan et al., 2004). In brief, adiponectin has both metabolic and vascular actions stimulating the endothelial production of $\mathrm{NO}$ and reduction in its levels may contribute to insulin resistance and endothelial/myocardial dysfunction (Dimmeler et al., 1999). Our results are also in keeping with the report that adiponectin is critical for endothelial cell survival and function (Ouchi et al., 2004) via the activation of eNOS and $\mathrm{pAKT}$ and $\mathrm{pAMPK}$ signaling.

In the normal heart, both fatty acid oxidation and glucose metabolism contribute to energy production. Conversely, the diabetic heart relies almost completely on fatty acids, as glucose uptake and subsequent utilization is impaired (Lopaschuk, 1996). There are several explanations for this switch in substrate utilization in diabetic heart. First, insulin-dependent glucose uptake, via the glucose transporter (GLUT) 4, is decreased, thus glucose metabolism (both glycolysis and glucose oxidation) is depressed. Second, the release of FFA from adipose tissue is increased. Despite an increase in FFA utilization in diabetic heart, it is likely that FFA uptake exceeds oxidation rate resulting in lipid accumulation in the myocytes and lipotoxicity (Sharma et al., 2004). Furthermore, lipid intermediates might promote apoptosis of cardiomyocytes, thus representing an additional mechanism leading to cardiac dysfunction. In order to explore the effect of adiponectin and HO-1 elevation on diabetic cardiac metabolism, we measured cardiac malonyl-CoA, a key mediator in the $\beta$-oxidation, due to its inhibitory effect on carnitine palmitoyltransferase 1 . With normal insulin sensitivity, malonyl-CoA increases when the muscle feeds on glucose (plasma insulin levels increased), while it decreases when the muscle is glucose deprived (plasma insulin levels decreased; Ruderman et al., 1999; Park et al., 2002). Insulin exerts its lipogenic effect increasing cellular malonyl-CoA via dephosphorylation and activation of acetylCoA carboxylase, thus supporting fatty acid synthesis instead of degradation. Hence, a decrease in insulin availability reduces intracellular malonyl-CoA levels and fatty acid oxidation increases. The present study confirms that the decreased insulin secretion (due to STZ injection) reduces malonyl-CoA formation. Following $\mathrm{CoPP}$ administration, $\mathrm{HO}-1$ induction reverses the decrease of malonyl-CoA observed in diabetic heart. The normalization of malonyl-CoA suggests a favorable effect on diabetic cardiac metabolism toward glucose utilization and prevention of cellular lipotoxicity (Figure 7). Since insulin sensitizing properties have been ascribed, to adiponectin, we speculate that malonyl-CoA-dependent decrease in $\beta$-oxidation might be due to adiponectin release or HO-1 induction. Anyway, further studies using adiponectin or HO-1 knockout mice are needed to explain the degree of involvement of these molecules in the control of $\beta$-oxidation rate in the heart.

Recently, it was reported that the effect of adiponectin on glucose uptake is mediated by $\mathrm{p} 38 \mathrm{MAPK}$-increasing GLUT4 translocation. However, adiponectin can also stimulate the translocation 


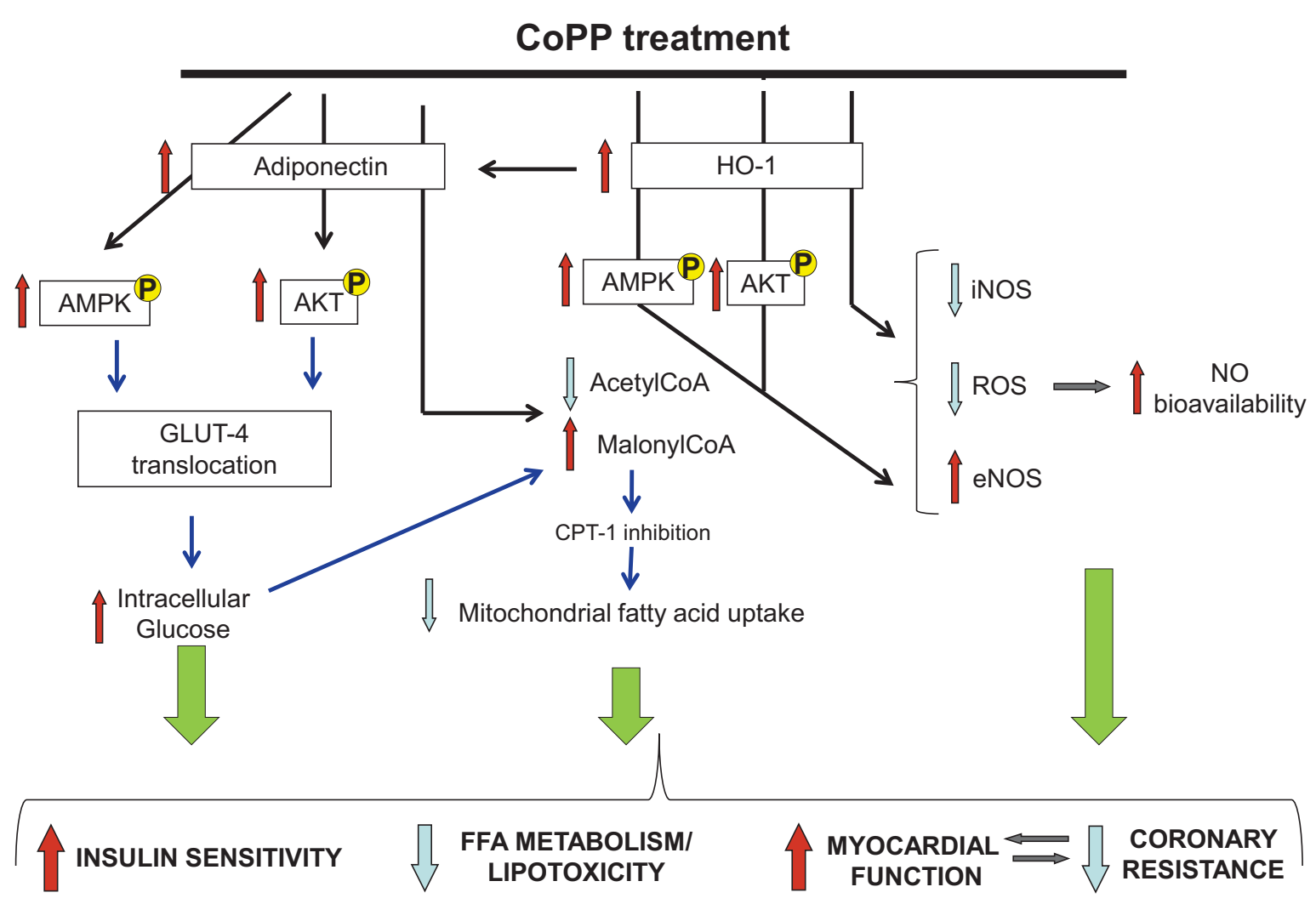

FIGURE 7 | Diagrammatic representation of the major changes observed in diabetic heart after CoPP treatment as compared to untreated hearts. Black arrows represent results demonstrated in this paper and, blue arrows represent previously shown work and/or hypothesis drawn from the literature.

of GLUT4 via PI-3-kinase, the same signaling cascade activated by insulin (Figure 7). Human studies demonstrate that plasma adiponectin and $\mathrm{HO}-1$ expression regulate insulin sensitivity and insulin receptor phosphorylation (Yamauchi et al., 2001; Stefan et al., 2004) contributing to increased insulin sensitivity. As a consequence, enhanced glucose utilization in CoPP-treated diabetic hearts could contribute toward a glucose-dependent energy repletion which in turn has the potential to enhance cellular malonyl-CoA. Together with improved myocardial perfusion and increased malonyl-CoA, reduced $\beta$-oxidation of FFA in myocardial cells could attenuate lipid peroxidation, oxidative stress, and improve myocytes function.

\section{REFERENCES}

Abraham, N. G., and Kappas, A. (2008). Pharmacological and clinical aspects of heme oxygenase. Pharmacol. Rev. 60, 79-127.

Adeghate, E. (2004). Molecular and cellular basis of the aetiology and management of diabetic cardiomyopathy: a short review. Mol. Cell. Biochem. 261, 187-191.

An, D., and Rodrigues, B. (2006). Role of changes in cardiac metabolism in development of diabetic cardiomyopathy. Am. J. Physiol. Heart Circ. Physiol. 291, H1489-H1506.

Antoniades, C., Antonopoulos, A. S., Tousoulis, D., and Stefanadis, C. (2009). Adiponectin: from obesity to cardiovascular disease. Obes. Rev. 10, 269-279.

Bardell, A. L., and MacLeod, K. M. (2001). Evidence for inducible nitric-oxide synthase expression and activity in vascular smooth muscle

Taken together, the results of the present study promote the use of compounds that increase HO-1 expression, HO activity, and adiponectin levels as a therapeutic means of both preventing and reversing the cardiomyopathy associated with diabetes.

\section{ACKNOWLEDGMENTS}

This work was supported by Scuola Superiore Sant'Anna and IFC-CNR research grants and by the American Heart Association (SGD to NP) and National Institutes of Health grants (HL55601, DK068134, and HL34300 to Nader G. Abraham; and HL075265 to NP).

of streptozotocin-diabetic rats. $J$ Pharmacol. Exp. Ther. 296, 252-259.

Boudina, S., and Abel, E. D. (2007). Diabetic cardiomyopathy revisited. Circulation 115, 3213-3223.

Boudina, S., and Abel, E. D. (2007). Diabetic cardiomyopathy revisited. Circulation 115, 3213-3223.

Ceriello, A., Quagliaro, L., D’Amico, M., Di Filippo, C.
Marfella, R., Nappo, F., Berrino, L., Rossi, F., and Giugliano, D. (2002). Acute hyperglycemia induces nitrotyrosine formation and apoptosis in perfused heart from rat. Diabetes 51, 1076-1082.

Chandran, M., Phillips, S. A., Ciaraldi, T., and Henry, R. R. (2003). Adiponectin: more than just another fat cell hormone? Diabetes Care 26, 2442-2450. 
Di Noia, M. A., Van Driesche, S., Palmieri, F., Yang, L. M., Quan, S., Goodman, A. I., and Abraham, N. G. (2006). Heme oxygenase-1 enhances renal mitochondrial transport carriers and cytochrome $\mathrm{C}$ oxidase activity in experimental diabetes. J. Biol. Chem. 281, 15687-15693.

Dimmeler, S., Fleming, I., Fisslthaler, B., Hermann, C., Busse, R., and Zeiher, A. M. (1999). Activation of nitric oxide synthase in endothelial cells by Akt-dependent phosphorylation. Nature 399, 601-605.

Ding, G., Qin, Q., He, N., Francis-David, S. C., Hou, J., Liu, J., Ricks, E., and Yang, Q. (2007). Adiponectin and its receptors are expressed in adult ventricular cardiomyocytes and upregulated by activation of peroxisome proliferator-activated receptor gamma. J. Mol. Cell. Cardiol. 43, 73-84.

Haidara, M. A., Yassin, H. Z., Rateb, M., Ammar, H., and Zorkani, M. A. (2006). Role of oxidative stress in development of cardiovascular complications in diabetes mellitus. Curr. Vasc. Pharmacol. 4, 215-227.

Hotta, K., Funahashi, T., Arita, Y., Takahashi, M., Matsuda, M., Okamoto, Y., Iwahashi, H., Kuriyama, H., Ouchi, N., Maeda, K., Nishida, M., Kihara, S., Sakai, N., Nakajima, T., Hasegawa, K., Muraguchi, M., Ohmoto, Y., Nakamura, T., Yamashita, S., Hanafusa, T., and Matsuzawa, Y. (2000). Plasma concentrations of a novel, adipose-specific protein, adiponectin, in type 2 diabetic patients. Arterioscler. Thromb. Vasc. Biol. 20, 1595-1599.

Kim, J. Y., van de, W. E., Laplante, M., Azzara, A., Trujillo, M. E., Hofmann, S. M., Schraw, T., Durand, J. L., Li, H., Li, G., Jelicks, L. A., Mehler, M. F., Hui, D. Y., Deshaies, Y., Shulman, G. I., Schwartz, G. J., and Scherer, P. E. (2007). Obesity-associated improvements in metabolic profile through expansion of adipose tissue. J. Clin. Invest. 117, 2621-2637.

Koch, M., Wendorf, M., Dendorfer, A., Wolfrum, S., Schulze, K., Spillmann, F., Schultheiss, H. P., and Tschope, C. (2003). Cardiac kinin level in experimental diabetes mellitus: role of kininases. Am. J. Physiol. Heart Circ. Physiol. 285, H418-H423.

Kusmic, C., L'Abbate, A., Sambuceti, G., Drummond, G., Barsanti, C., Matteucci, M., Cao, J., Piccolomini, F., Cheng, J., and Abraham, N. G.
(2010). Improved myocardial perfusion in chronic diabetic mice by the up-regulation of pLKB1 and AMPK signaling. J. Cell. Biochem. 109, 1033-1044.

L'Abbate, A., Neglia, D., Vecoli, C., Novelli, M., Ottaviano, V., Baldi, S., Barsacchi, R., Paolicchi, A., Masiello, P., Drummond, G. S., McClung, J. A., and Abraham, N. G. (2007). Beneficial effect of heme oxygenase-1 expression on myocardial ischemia-reperfusion involves an increase in adiponectin in mildly diabetic rats. Am. J. Physiol. Heart Circ. Physiol.293, H3532-H3541.

Lazzarino, G., Amorini, A. M., Fazzina, G., Vagnozzi, R., Signoretti, S., Donzelli, S., Di Stasio, E., Giardina, B., and Tavazzi, B. (2003). Singlesample preparation for simultaneous cellular redox and energy state determination. Anal. Biochem. 322, 51-59.

LeWinter, M. M. (1996). Diabetic cardiomyopathy: an overview. Coron. Artery Dis. 7, 95-98.

Li, M., Kim, D. H., Tsenovoy, P. L., Peterson, S. J., Rezzani, R., Rodella, L. F., Aronow, W. S., Ikehara, S., and Abraham, N. G. (2008). Treatment of obese diabetic mice with a heme oxygenase inducer reduces visceral and subcutaneous adiposity, increases adiponectin levels, and improves insulin sensitivity and glucose tolerance. Diabetes 57, 1526-1535.

Lopaschuk, G. D. (1996). Abnormal mechanical function in diabetes: relationship to altered myocardial carbohydrate/lipid metabolism. Coron. Artery Dis. 7, 116-123.

Nagareddy, P. R., Xia, Z., McNeill, J. H., and MacLeod, K. M. (2005). Increased expression of iNOS is associated with endothelial dysfunction and impaired pressor responsiveness in streptozotocin-induced diabetes. Am. J. Physiol. Heart Circ. Physiol. 289, H2144-H2152.

Ouchi, N., Kobayashi, H., Kihara, S., Kumada, M., Sato, K., Inoue, T., Funahashi, T., and Walsh, K. (2004). Adiponectin stimulates angiogenesis by promoting crosstalk between AMP-activated protein kinase and Akt signaling in endothelial cells. J. Biol. Chem. 279, 1304-1309.

Pacher, P., Liaudet, L., Bai, P., Mabley, J. G., Kaminski, P. M., Virag, L., Deb, A., Szabo, E., Ungvari, Z., Wolin, M. S., Groves, J. T., and Szabo, C. (2003). Potent metalloporphyrin peroxynitrite decomposition catalyst protects against the development of doxorubicin-induced cardiac dysfunction. Circulation 107, 896-904.

Park, H., Kaushik, V. K., Constant, S., Prentki, M., Przybytkowski, E., Ruderman, N. B., and Saha, A. K. (2002). Coordinate regulation of malonyl-CoA decarboxylase, sn-glycerol-3-phosphate acyltransferase, and acetyl-CoA carboxylase by AMP-activated protein kinase in rat tissues in response to exercise. $J$. Biol. Chem. 277, 32571-32577.

Ruderman, N. B., Saha, A. K., Vavvas, D. and Witters, L. A. (1999). MalonylCoA, fuel sensing, and insulin resistance. Am. J. Physiol. 276, E1-E18.

Sharma, S., Adrogue, J. V., Golfman, L., Uray, I., Lemm, J., Youker, K., Noon, G. P., Frazier, O. H., and Taegtmeyer, H. (2004). Intramyocardial lipid accumulation in the failing human heart resembles the lipotoxic rat heart. FASEB J. 18, 1692-1700.

Stefan, N., Vozarova, B., Funahashi, T. Matsuzawa, Y., Weyer, C., Lindsay, R. S., Youngren, J. F., Havel, P. J., Pratley, R. E., Bogardus, C., and Tataranni, P. A. (2004). Plasma adiponectin concentration is associated with skeletal muscle insulin receptor tyrosine phosphorylation, and low plasma concentration precedes a decrease in whole-body insulin sensitivity in humans. Diabetes 18, 1692-1700.

Stevens, M. J. (2005). Oxidativenitrosative stress as a contributing factor to cardiovascular disease in subjects with diabetes. Curr. Vasc. Pharmacol. 3, 253-266.

Szabo, C., Mabley, J. G., Moeller, S. M., Shimanovich, R., Pacher, P., Virag, L., Soriano, F. G., Van Duzer, J. H., Williams, W., Salzman, A. L., and Groves, J. T. (2002). Part I: pathogenetic role of peroxynitrite in the development of diabetes and diabetic vascular complications: studies with FP15, a novel potent peroxynitrite decomposition catalyst. Mol. Med. 8, 571-580.

Tao, L., Gao, E., Jiao, X., Yuan, Y., Li, S., Christopher, T. A., Lopez, B. L., Koch, W., Chan, L., Goldstein, B. J., and Ma, X. L. (2007). Adiponectin cardioprotection after myocardial ischemia/reperfusion involves the reduction of oxidative/nitrative stress. Circulation 115, 1408-1416.

Turkseven, S., Kruger, A., Mingone, C. J., Kaminski, P., Inaba, M., Rodella, L. F., Ikehara, S., Wolin, M. S., and Abraham, N. G. (2005). Antioxidant mechanism of heme oxygenase-1 involves an increase in superoxide dismutase and catalase in experimental diabetes. Am. J. Physiol. Heart Circ. Physiol. 289, H701-H707.

Weinstein, D. M., Mihm, M. J., and Bauer, J. A. (2000). Cardiac peroxynitrite formation and left ventricular dysfunction following doxorubicin treatment in mice. $J$. Pharmacol. Exp. Ther. 294, 396-401.

Weyer, C., Funahashi, T., Tanaka, S., Hotta, K., Matsuzawa, Y., Pratley, R. E., and Tataranni, P. A. (2001). Hypoadiponectinemia in obesity and type 2 diabetes: close association with insulin resistance and hyperinsulinemia. J. Clin. Endocrinol. Metab. 86, 1930-1935

Yamauchi, T., Kamon, J., Waki, H., Terauchi, Y., Kubota, N., Hara, K., Mori, Y., Ide, T., Murakami, K., Tsuboyama-Kasaoka, N., Ezaki, O., Akanuma, Y., Gavrilova, O., Vinson, C., Reitman, M. L., Kagechika, H., Shudo, K., Yoda, M., Nakano, Y., Tobe, K., Nagai, R., Kimura, S., Tomita, M., Froguel, P., and Kadowaki, T. (2001). The fat-derived hormone adiponectin reverses insulin resistance associated with both lipoatrophy and obesity. Nat. Med. 7, 941-946.

Conflict of Interest Statement: The authors declare that the research was conducted in the absence of any commercial or financial relationships that could be construed as a potential conflict of interest.

Received: 29 February 2012; paper pending published: 09 April 2012; accepted: 07 May 2012; published online: 04 June 2012.

Citation: Cao J, Vecoli C, Neglia D, Tavazzi B, Lazzarino G, Novelli $M$, Masiello P, Wang Y-t, Puri N, Paolocci N, L'Abbate A and Abraham NG (2012) Cobalt-protoporphyrin improves heart function by blunting oxidative stress and restoring NO synthase equilibrium in an animal model of experimental diabetes. Front. Physio. 3:160. doi: 10.3389/fphys.2012.00160

This article was submitted to Frontiers in Fatty Acid and Lipid Physiology, a specialty of Frontiers in Physiology. Copyright (c) 2012 Cao, Vecoli, Neglia, Tavazzi, Lazzarino, Novelli, Masiello, Wang, Puri, Paolocci, L'Abbate and Abraham. This is an open-access article distributed under the terms of the Creative Commons Attribution Non Commercial License, which permits noncommercial use, distribution, and reproduction in other forums, provided the original authors and source are credited. 